\title{
The Use of Theory to Develop Physical Activity Interventions in Urological Cancer Survivors: A Narrative Review
}

\author{
Elke Rammant, MSc ${ }^{\mathrm{a}, *}$, Renée Bultijnck, MSc ${ }^{\mathrm{a}, \mathrm{b}}$, Cristina M. Caperchione, $\mathrm{PhD}^{\mathrm{c}}$, \\ Linda Trinh, $\mathrm{PhD}^{\mathrm{d}}$, on behalf of the research collaboration group of the cancer prevention and \\ management Special Interest Group of ISBNPA \\ ${ }^{a}$ Ghent University, Human Structure and Repair, Ghent, Belgium \\ ${ }^{\mathrm{b}}$ Research Foundation, Flanders (FWO), Brussels, Belgium \\ ${ }^{\mathrm{c}}$ Faculty of Health, Human Performance Research Centre, University of Technology Sydney, Sydney, New South Wales, Australia \\ ${ }^{\mathrm{d}}$ Faculty of Kinesiology and Physical Education, University of Toronto, Toronto, Canada
}

\section{A R T I C L E I N F O}

\section{Keywords:}

Urological cancer

Behavior change

Theory

Physical activity

Exercise

Intervention mapping

\begin{abstract}
A B S T R A C T
Objectives: To summarize the current available evidence on the use of behavior change theories to explain and change physical activity behavior in urological cancer survivors.

Data Sources: Five electronic databases including Medline, Web of Science, Embase, Cochrane, and Psych INFO and reference lists of key studies were searched between database inception and November 2020.

Peer-reviewed articles on the use of behavior change theories to understand or change physical activity in urological cancer survivors were included.

Conclusion: The theory of planned behavior and the social cognitive theory were the most used theories to explain and change physical activity behavior in urological cancers, respectively. However, the use of behavior change theories in physical activity interventions for urological cancers is still low across all urological tumor groups. Planning frameworks such as the intervention mapping approach should be used to enhance the systematic use of behavior change theories during every phase of intervention development. In addition, more research is needed to identity which behavior change techniques are most effective to change physical activity behavior in urological cancer survivors.

Implications for Nursing Practice: Nurses play a key role in the urological cancer patients' clinical pathway and should be able to motivate patients to engage in sufficient physical activity levels. Therefore, it is important that nurses understand the underlying reasons why patients (do not) engage in physical activity and which behavior change techniques are most effective in changing a patients' behavior.
\end{abstract}

(C) 2020 Elsevier Inc. All rights reserved.

\section{Introduction}

\section{Burden of Urological Cancers}

The incidence in the Western world of urological cancers is high with prostate, bladder, and kidney cancer being the most common. ${ }^{1}$ In addition, survival rates for those living with urological cancers continues to increase due to advances in diagnosis and treatment. ${ }^{2}$ Urological cancer survivors are generally diagnosed at an advanced age and have multiple comorbidities. ${ }^{3}$

One of the primary treatment options in this population is surgery, which is complex and associated with moderate to high complication

\footnotetext{
* Address correspondence to: Elke Rammant, MSc. Department of Human Structure and Repair, Ghent University, Corneel Heymanslaan 10, B-9000 Ghent, Belgium. Phone: +32-9-33-21552

E-mail address: elke.rammant@ugent.be (E. Rammant).
}

rates, depending on the type of surgery (ie, cystectomy 56\%, nephrectomy $21 \%$, prostatectomy $19 \%) .{ }^{4}$ Other frequently experienced problems after surgery are fatigue, diminished physical function and treatment-related symptoms leading to a decrease in health-related quality of life (HRQoL). ${ }^{5}$ Some of the most common treatment-related symptoms after surgery but also after radical radiotherapy are urinary, gastro-intestinal and/or sexual dysfunctions, ${ }^{6,7}$ which may in turn increase the risk of mental health issues such as depression, anxiety and stress. ${ }^{8}$ Mental health issues are also common in patients with urological cancer at diagnosis, which may independently worsen patient morbidity and mortality rates. ${ }^{9,10}$

In addition, almost half of patients with prostate cancer receive androgen deprivation therapy (ADT) at some point in their treatment. ADT leads to a variety of side effects with changes in body composition (ie, decrease in bone mineral density and muscle mass, increase in fat mass), fatigue, sexual dysfunction, and hot flushes as most common side effects. ${ }^{11}$ These complications and side effects, combined 
TABLE 1

Overview of PA Patterns in Urological Cancer Patients/Survivors

\begin{tabular}{|c|c|c|c|c|}
\hline First Author, year & Tumor group & No. of patients & Type of Measurement & Meeting PA guidelines \\
\hline Galvao et al, $2015^{29}$ & PCa patients & 463 & GLTEQ & $\begin{array}{l}12.3 \% \text { sufficient active } \\
40.2 \% \text { insufficiently active } \\
47.5 \% \text { inactive }\end{array}$ \\
\hline Blanchard et al, $2008^{31}$ & $\begin{array}{l}\text { Mixed group of patients with breast, } \\
\text { prostate, colorectal, bladder, uter- } \\
\text { ine and skin cancer }\end{array}$ & $\begin{array}{l}\text { Total } 9105 \text {, of which } 2226 \\
\text { prostate and } 586 \text { bladder }\end{array}$ & GLTEQ & $\begin{array}{l}\text { Prostate cancer patients; } \\
\text { 43.2\% meeting PA recommendations } \\
\text { Bladder cancer patients; } \\
36.0 \% \text { meeting PA recommendations }\end{array}$ \\
\hline Gopalakrishna et al, $2017^{33}$ & $\mathrm{BC}$ patients & 472 & IPAQ & $\begin{array}{l}\text { 50\% high PA levels } \\
\text { 26\% median PA level } \\
\text { 24\% low PA level }\end{array}$ \\
\hline Trinh et al, $2018^{34}$ & Kidney cancer survivors & 703 & Modified GLTEQ & $\begin{array}{l}10.1 \% \text { combined exercise guidelines } \\
15.9 \% \text { aerobic-only guideline } \\
8.8 \% \text { strength-only guideline } \\
65.1 \% \text { none of the exercise guidelines }\end{array}$ \\
\hline
\end{tabular}

Abbreviations: PA, physical activity; PCa, prostate cancer; BC, bladder cancer; GLTEQ Godin Leisure Time Exercise Questionnaire; IPAQ International Physical Activity Questionnaire.

with their advanced age, contribute to a decline in physical function and decrease in HRQoL. ${ }^{11}$

Therefore, holistic supportive care interventions are needed to reduce the chronic and late effects of cancer treatment. Physical activity (PA) has a positive affect on clinical outcomes such as improvement in HRQoL, cancer-specific mortality, and reducing treatment-related toxicities across many cancer survivor groups, ${ }^{12}$ including urological cancer survivors. ${ }^{13}$

\section{Effects of Exercise on Symptom Management}

Engaging in sufficient levels of PA or structured exercise is a relatively inexpensive and safe strategy to mitigate treatment-related side effects or improve different aspects of HRQoL. ${ }^{14}$ Further, some research has revealed contrasting evidence; however, this research mainly reported on breast cancer, was focused on multimodal treatments and included only a small number of studies. ${ }^{15}$

Several studies investigated the effects of exercise in men with prostate cancer and showed beneficial results in outcomes such as fatigue, physical fitness, body composition parameters, anxiety and depression, HRQoL, comorbidities, risk of recurrence, and cancer-specific survival. ${ }^{11,16,17}$ Most evidence for these beneficial effects were found for supervised PA programs with moderate-intensity combined aerobic plus resistance training sessions performed two to three times per week or twice-weekly moderate-intensity resistance training for at least 12 weeks. ${ }^{18,19}$

Emerging evidence exists that describes positive effects of exercise on sexual desire and sexual activity in men with prostate cancer. ${ }^{20}$ Patients with prostate cancer who engage in sufficient PA also showed lower prostate-specific antigen levels, delay in initiating ADT by 2 years, lower serum insulin and insulin-like growth factor (IGF1), higher IGF binding protein (IGFBP-1), and a lower risk of highgrade disease (Gleason score 7 or greater) compared with less-active patients. ${ }^{13}$

Few PA studies have been conducted in bladder and kidney cancer survivors. However, a systematic review in patients with bladder cancer (including 3 exercise studies) showed that exercise training has the potential to improve muscle strength, activities of daily living, physical fitness, and some HRQoL domains. ${ }^{21}$ For kidney cancer, positive associations were found between PA and HRQoL. ${ }^{22}$ A recent systematic review also suggested beneficial effects of exercise on mortality in bladder and kidney cancer. ${ }^{17}$
In patients undergoing major cancer surgery with additional (neo) adjuvant treatments such as chemotherapy and/or radiotherapy, it has been demonstrated that PA can play an important role in the preoperative setting (also known as "prehabilitation"). Research shows that prehabilitation interventions aimed at increasing PA, can improve the physical and mental functioning of the patient. ${ }^{21,23}$ For instance, it can facilitate the return to the highest possible PA level and provide an opportunity to become immediately involved in their own care.

\section{Patterns of Physical Activity in Urological Cancers}

Studies showed that cancer survivors are interested in receiving PA advice from their health care team. ${ }^{24}$ However, only a small minority (on average $18.3 \%{ }^{25}$ ) of survivors are receiving a referral to an exercise program. ${ }^{25-28}$

The updated guidelines of 2019 for cancer survivors recommend thrice-weekly aerobic activity for 30 minutes and twice-weekly resistance exercise ( 1 exercise per major muscle group, $8-15$ repetitions per set, 2 sets per exercise), progressing with small increments. ${ }^{18}$ However, a majority of urological patients are not meeting these PA guidelines. Table 1 shows an overview of the PA patterns in urological patients.

The beneficial effects of PA and exercise for urological cancer survivors are clear, however, only the minority of the survivors are reaching sufficient PA levels. To achieve PA behavior change, psychological theories of motivation and behavior change should be used to address the barriers and facilitators of PA experienced by the patients. ${ }^{35} \mathrm{~A}$ variety of theories exist that explain the mechanisms (ie, cognitive, affective, and behavioral) through which behavior can be targeted. ${ }^{35}$ Theoretical approaches are also needed to identify key motivational outcomes to facilitate the adoption and maintenance of PA. ${ }^{36}$

The aim of this study is to summarize the current available evidence on the use of behavior change theories to explain and change PA behavior in urological cancer survivors.

\section{Methods}

A narrative review was conducted of peer-reviewed English language literature published from inception until November 2020. Our population-of-interest was urological cancer survivors. Only prostate, bladder, and kidney cancer were included as these are the three most common urological cancers. Both randomized controlled trails and 
non-randomized studies (ie, cohort studies, case-control studies, longitudinal studies, and cross-sectional studies) were considered for inclusion. Furthermore, also systematic reviews were considered. Studies that explained or changed physical activity using behavior change theories were considered relevant. Following search teams were used; "physical activity/exercise", "prostate, bladder and/or kidney cancer", and keywords that are associated with behavior change theories (behavior change, behavior change techniques, form of delivery, motivation, theories, facilitators and barriers, planning frameworks).

Data sources used for this review were electronic databases including Medline, Web of Science, Embase, Cochrane, and Psych INFO and reference lists of key studies.

\section{Results}

Growing evidence shows that theory-based behavior change interventions are more likely to succeed and to sustain, than non-theoretical interventions. ${ }^{37,38}$ This trend has also been conveyed in cancer-specific PA research. ${ }^{39}$ Theories can help identify the behavioral constructs that should be targeted, the mechanisms underpinning the "active ingredients" (BCTs) used to change behavior, and how those constructs are interconnected. ${ }^{40,41}$ Theory-based behavior change interventions are also able to provide information on how or why an intervention works, which increases the utility of the results of an intervention. ${ }^{37}$ Behavior change interventions, guided by theory, provide a framework to systematically develop and evaluate interventions, and should include three broad components: (1) theories to explain behavior, (2) BCTs to change behavior, and (3) form of delivery. ${ }^{40,41}$

\section{Explaining Physical Activity Behavior in Patients With Urological Cancer}

Meeting PA guidelines can be challenging for patients undergoing urological cancer treatments and underlying reasons or determinants for this can be multifactorial. Research showed that disease, treatment-related factors and a numerous of demographic factors, such as age or socioeconomic background, are related to the likelihood of a patient being physically active. ${ }^{42}$ Although we cannot change these factors, they do help explain why an intervention is (not) working. We can however have an effect on the modifiable factors at an individual or environmental level, to influence patient's PA behavior.

The Theory of Planned Behavior (TPB $)^{43}$ has been the most tested theory to date in patients with cancer to explain PA behavior. ${ }^{44}$ The TPB states that intention to perform a particular behavior is the primary determinant of behavior. Intention, in turn, is determined by three other constructs: subjective norm, attitude, and perceived behavior control. Each of these constructs is considered a higherorder structure composed of two lower-order components. The lower-order components of subjective norm are descriptive (perception that important others exercise) and injunctive (perception that important others approve of exercise) components. Attitude consists of affective (enjoyment of exercise) and instrumental (perceived benefits of exercise) components, and perceived behavior control includes self-efficacy (confidence in ability to exercise) and perceived control (perceived control over exercise). ${ }^{43}$

A study in patients with prostate cancer found that perceived behavioral control was the only determinant of exercise intention in these survivors, explaining $36 \%$ of the variance in exercise behavior. In bladder cancer survivors, exercise behavior was predicted by perceived behavior control whereas exercise intention was predicted by affective attitude, instrumental attitude, descriptive norm, and perceived behavior control. In kidney cancer survivors, all constructs of the TPB, except for injunctive norm and affective attitude, explained exercise intention with perceived behavior control as most important construct. $^{45}$
In addition to the TPB, other common behavior change theories have been used to help explain PA behavior of urological cancer survivors. In a study by Courneya et $\mathrm{al}^{46}$ stages of change (as described in The Transtheoretical Model[TTM]) was found to be a strong predictor of exercise adherence in patients with prostate cancer. ${ }^{46}$ The TTM states that an individual goes through different stages of readiness to make behavioral changes and therefore require interventions tailored to the patient individual stage. The five stages of change are as follows: precontemplation (ie, no intention of becoming physically active in the next 6 months), contemplation (ie, intending to become physically active within the next 6 months), preparation (ie, making small changes in behavior but still not meeting a criterion for PA), action (ie, meeting a criterion of PA for $<6$ months), and maintenance (ie, meeting a criterion for PA for $\geq 6$ months). In addition, the TTM identified 10 processes of change along with decisional balance, selfefficacy, and temptations as important constructs for behavior change. In the earlier stages of change emphasizing experiential or cognitive processes of change are recommended (eg, understanding the risks of low levels of PA), whereas in later stages of change promoting behavioral processes of change are more indicated (eg, rewarding one-self). ${ }^{47,48}$

A study in bladder cancer survivors used a qualitative approach to examine factors related to PA behavior. ${ }^{49}$ This study identified demographical, disease, treatment, and psychological factors, as well as social, health system, and environmental factors, to explain PA behavior in bladder cancer survivors. ${ }^{49}$ The results indicated that PA behavior should also be explained by using social ecological models in addition to individual factors including social (eg, social support), policy (e.g. reimbursements, rehabilitation programs) and environmental factors (eg, weather, distance to exercise facilities). However, no quantitative study to date has tested social ecological models in bladder cancer survivors. In kidney cancer, one study examined which social ecological factors are related to PA and found that only perceived proximity of retail shops was a significant predictor of meeting PA guidelines. ${ }^{50}$

\section{Changing Behavior in Patients With Urological Cancer}

A recent systematic review in prostate cancer evaluated PA-based behavior change interventions and identified six prostate cancer and six mixed cancer studies of which only three prostate cancer and five mixed cancer studies incorporated theories in their interventions. In the prostate cancer only studies, one study used Social Cognitive Theory (SCT), one study the TPB, and another study was grounded in multiple theories, including the SCT and the TTM. All of the mixed cancer studies incorporated multiple theories including the SCT, the TTM, the TPB, the social ecological model and the chronic disease self-management framework. ${ }^{51}$

Bandura's SCT is a theory that is often used for both explaining and changing behavior. The determinants described by the SCT are outcome expectations, self-efficacy, behavioral capability, perceived behavior of others, and environment. The SCT integrates its determinants of behavior with specific BCTs such as active learning, reinforcement, enactive mastery experiences, modeling, guided practice, verbal persuasion, improving physical and emotional states, and facilitation. $^{52,53}$

A majority of the studies in the systematic review by Finlay et $\mathrm{al}^{51}$ used a combination of BCTs with goal setting, encouragement to selfmonitor, provision of information about the consequences of the behavior, and barrier identification as the most common. Social support, through supervised programs with social group interactions and demonstration of behavior, was more common in prostate cancer only studies compared with mixed cancer studies. ${ }^{51}$

Another framework identified during the search is the Multi-Process Action Control framework (M-PAC), which was recently used in a physical activity pilot study in prostate cancer. ${ }^{54}$ The M-PAC 
framework aims to reduce the intention-behavior gap and focuses on translating intentions into actions, developing PA habits (ie, cuebased reminders) and forming an exercise identity. ${ }^{55}$ The study by Trinh et $\mathrm{al}^{54}$ showed the utility of the M-PAC to guide and understand behavior change in prostate cancer survivors.

A recent systematic review in patients with prostate cancer also identified and evaluated BCTs in the included studies. ${ }^{56}$ The most common BCTs identified were behavioral practice/rehearsal, instruction on how to perform the behavior, self-monitoring of behavior, adding objects to the environment, social support (unspecified), and generalization of target behavior. However, evaluation of the BCTs did not show any difference in efficacy between the number and types of BCTs. Some evidence suggested that BCTs that were well taught and explained to the cancer survivors were more effective than BCTs that were less well explained to the patients. ${ }^{56}$

For bladder cancer, only one PA intervention is presented in the literature that is developed based on a behavior change theory. ${ }^{57}$ Another study in patients with bladder cancer described the use of $\mathrm{BCT}$ in their intervention, although not referring to the term "ВСТ" ${ }^{58}$ The strategies used in the study by Jensen et $\mathrm{al}^{58}$ are "set graded tasks" and "prompt self-monitoring of behavior". For kidney cancer, one behavior change intervention based its counseling strategies on the TPB. This study showed preliminary evidence that adding behavioral counseling (based on the TPB) improved PA, physical functioning, and short-term motivation, and self-regulatory outcomes in kidney cancer survivors. ${ }^{59,60}$

\section{Form of Delivery of Behavior Change Interventions}

Although less commonly discussed in the literature, form of delivery is next to theory and BCTs an important ingredient for behavior change interventions. The form of delivery describes the ways in which an intervention is delivered. The form of delivery of an intervention includes different components such as the provider, format, materials, setting, intensity, tailoring, and style. ${ }^{40,41}$ Form of delivery is important for several reasons such as operationalizing theories into concrete intervention components, enhancing or undermining BCT effectiveness, influencing intervention engagement, adherence, and fidelity, determining how users understand intervention content, influencing effectiveness beyond the BCT, and for implementation and sustainability. ${ }^{40}$

\section{Discussion}

Framework for Developing Theory- and Evidence-Based Behavior Change Interventions

The use of theory to develop PA interventions is still scarce. Furthermore, adequate descriptions of the theories that were used in the interventions are needed to identify the "active components" of successful interventions and to expand and test the evidence across settings and facilitate evidence synthesis. Next to the use of theory, also other resources (eg, literature review, collection of new data, involvement of stakeholders) are required to develop behavior change interventions. Hence, this might be challenging and frameworks to guide this development process are needed. Therefore, planning frameworks exist that guide the systematic development of theory- and evidence-based behavior change interventions.

An example of a planning framework is the intervention mapping (IM) protocol. ${ }^{61}$ This is a detailed protocol for the planning and development of theory- and evidence-based health promotion interventions (in this case increasing PA levels). The IM approach is characterized by three perspectives applied during the program planning process; (1) it takes an ecological and systemic approach to understand health problems and consequently intervene at multiple levels to address them (ie, individual, interpersonal, organizational and community), (2) it is grounded in community-based participatory research methods to ensure that the interventions match the specific priorities of the needs and context of the population, and (3) the eclectic use of theory. The IM protocol exists of a systematic development process of intervention planning in six steps: (1) conduct a needs assessment, (2) create matrices of change objectives, (3) select theory-based intervention methods an practical applications, (4) organize methods and applications into an intervention program, (5) plan for adoption, implementation and sustainability of the program, and (6) generate an evaluation plan. ${ }^{62}$

Another planning framework is the Medical Research Council framework, published in 2000 and updated in $2008 .^{63}$ The goal is to assist researchers to recognize and adopt appropriate methods in the process of developing and evaluating complex interventions. The framework follows several phases, not necessarily in a linear sequence. The key elements are; developing an intervention (ie, identifying the evidence base, identifying or developing theory, and modeling process and outcomes), piloting and feasibility (ie, testing procedures, estimating recruitment and retention, and determining sample size), evaluating the intervention (ie, assessing effectiveness, understanding change process, and assessing cost effectiveness), and implementation (ie, dissemination, surveillance and monitoring, and long-term follow-up).

These planning frameworks and others have been used across various disciplines, but the use of it in urological PA cancer research is rare. Only a few studies in urological cancers are using a planning framework to develop and test their PA behavior change intervention. For example, OncoActive in prostate cancer, ${ }^{64}$ ExerciseGuide in metastatic prostate cancer, ${ }^{65}$ and The POPEYE trial in bladder cancer. ${ }^{66}$ Although these planning frameworks are not the only way to develop behavior change interventions, it might help researchers to develop and evaluate their intervention with a systematic approach.

\section{Implications for Nursing Practice}

For future intervention development, we would strongly recommend using a planning framework, although we acknowledge that this is a time-consuming event. Furthermore, we recommend considering the use of a combination of theories for both explaining and changing PA behavior rather than trying to fit everything in one specific theory, to allow synergistic effects and enhance intervention effectiveness. ${ }^{67}$

To increase evidence synthesis and identification of the effective ingredients within interventions, it is also crucial to thoroughly report the used theories and BCTs. Often studies report results without adding detailed information about the development of the intervention. It is important to state that also interventions that did not follow an in-depth planning framework approach should report the BCTs used and, if possible, include additional details regarding the dose, frequency, and quality of implementation of the BCTs. To increase consistency in reporting BCTs, we advise to use a classification model such as, the Coventry, Aberdeen, and London-Refined (CALO-RE) taxonomy of behavior change techniques. Furthermore, this method will enable in the search for psychological mediators that reveal why interventions (do not) work. ${ }^{68}$ Specific attention is also indicated in the specification of the type of BCT per tumor group/ disease stage and treatment, to further tailor interventions within the cancer survivorship population.

Another reporting gap is that primarily short-term data are published. Therefore, future studies should implement longer-term follow-up for physical activity maintenance, which is particularly important for long-term outcomes in urological cancer survivors.

Finally, physical activity interventions should focus on understanding mediating mechanisms to uncover "what worked" and "what did not work" in a behavior change intervention. ${ }^{69}$ Mediators are intervening causal variables that are needed to complete a 
cause-effect pathway between an intervention and physical activity. ${ }^{70}$ Knowledge of mediators will assist with effective design of interventions and targeting key constructs that are needed for behavior change. ${ }^{71}$ Physical activity theories suggest that specific constructs are critical antecedents of engaging in physical activity. These constructs are hypothesized as components of a causal chain where if the mediators are changed, a change in physical activity should follow. ${ }^{72}$ However, few behavior change interventions examine the mechanisms of change, and therefore pilot studies that demonstrate change in the proposed mediators are needed before larger-scale randomized controlled trials.

\section{Conclusion}

The use of behavior change theories in physical activity interventions for urological cancers is limited across all urological tumor groups. For optimal long-term health benefits, cancer survivors need to be continually active throughout the cancer care continuum. Nurses play a key role in the urological patients' clinical pathway and should be able to motivate patients to engage in sufficient physical activity levels. Therefore, it is important that nurses understand the underlying reasons why patients (do not) engage in physical activity, and which behavior change techniques are most effective in changing a patient's behavior.

Planning frameworks such as the intervention mapping approach should be used to enhance the systematic use of behavior change theories during every phase of intervention development. In addition, future research is needed to identify which behavior change techniques are most effective to change physical activity behavior in urological cancer survivors.

\section{Disclosures}

This research did not receive any specific grant from funding agencies in the public, commercial, or not-for-profit sectors.

\section{References}

1. Hoffman A, Half. Update on screening for urological malignancies. Rambam Maimonides Med J. 2017;8:e041.

2. Dy GW, Gore JL, Forouzanfar MH, Naghavi M, Fitzmaurice CJEu. Global burden of urologic cancers, 1990-2013. Eur Urol. 2017;71:437-446.

3. Garg T, Young AJ, Kost KA, et al. Burden of multiple chronic conditions among patients with urological cancer. J Urol. 2018;199:543-550.

4. Patel HD, Ball MW, Cohen JE, Kates M, Pierorazio PM, Allaf MEJU. Morbidity of urologic surgical procedures: an analysis of rates, risk factors, and outcomes. Urology. 2015;85:552-560

5. Sanda MG, Dunn RL, Michalski J, et al. Quality of life and satisfaction with outcome among prostate-cancer survivors. N Engl J Med. 2008;358:1250-1261.

6. Rammant E, Van Wilder L, Van Hemelrijck M, et al. Health-related quality of life overview after different curative treatment options in muscle-invasive bladder cancer: an umbrella review. Qual Life Res. 2020;29:2887-2910.

7. Michaelson MD, Cotter SE, Gargollo PC, Zietman AL, Dahl DM, Smith MR. Management of complications of prostate cancer treatment. CA Cancer J Clin. 2008;58:196-213

8. Pham H, Torres H, Sharma P. Mental health implications in bladder cancer patients: a review. Urol Oncol. 2019;37:97-107.

9. van Stam MA, van der Poel HG, Bosch JR, et al. Prevalence and correlates of mental health problems in prostate cancer survivors: a case-control study comparing survivors with general population peers. Urol Oncol. 2017;35. 531. e531-531. e537.

10. Watts S, Leydon G, Birch B, et al. Depression and anxiety in prostate cancer: a systematic review and meta-analysis of prevalence rates. BMJ Open. 2014;4: e003901.

11. Nguyen PL, Alibhai SM, Basaria S, et al. Adverse effects of androgen deprivation therapy and strategies to mitigate them. Eur Urol. 2015;67:825-836.

12. Mishra SI, Scherer RW, Geigle PM, et al. Exercise interventions on health-related quality of life for cancer survivors. Cochrane Database Syst Rev. 2012;8: CD007566.

13. Keogh JW, MacLeod RD. Body composition, physical fitness, functional performance, quality of life, and fatigue benefits of exercise for prostate cancer patients: a systematic review. J Pain Symptom Manage. 2012;43:96-110.

14. Heywood R, McCarthy AL, Skinner TL. Safety and feasibility of exercise interventions in patients with advanced cancer: a systematic review. Support Care Cancer. 2017;25:3031-3050.
15. Loughney LA, West MA, Kemp GJ, Grocott MP, Jack S. Exercise interventions for people undergoing multimodal cancer treatment that includes surgery. Cochrane Database Syst Rev. 2018;12: CD012280.

16. Gardner JR, Livingston PM, Fraser SFJJCO. Effects of exercise on treatment-related adverse effects for patients with prostate cancer receiving androgen-deprivation therapy: a systematic review. J Clin Oncol. 2014;32:335-346.

17. Friedenreich CM, Stone CR, Cheung WY, Hayes SC. Physical activity and mortality in cancer survivors: a systematic review and meta-analysis. JNCI Cancer Spectrum. 2020;4:pkz080.

18. Campbell KL, Winters-Stone KM, Wiskemann J, et al. Exercise guidelines for cancer survivors: consensus statement from international multidisciplinary roundtable. MedSci Sports Exerc. 2019;51:2375-2390.

19. Bourke L, Gilbert S, Hooper R, et al. Lifestyle changes for improving disease-specific quality of life in sedentary men on long-term androgen-deprivation therapy for advanced prostate cancer: a randomised controlled trial. Eur Urol. 2014;65:865-872.

20. Cormie P, Newton RU, Taaffe DR, Spry N, Galvão DA. Exercise therapy for sexual dysfunction after prostate cancer. Nat Rev Urol. 2013;10:731-736.

21. Rammant E, Decaestecker K, Bultijnck R, et al. A systematic review of exercise and psychosocial rehabilitation interventions to improve health-related outcomes in patients with bladder cancer undergoing radical cystectomy. Clin Rehab. 2018;32:594-606

22. Trinh L, Plotnikoff RC, Rhodes RE, North S, Courneya KS, Biomarkers P. Associations between physical activity and quality of life in a population-based sample of kidney cancer survivors. Cancer Epidemiol Biomarkers Prev. 2011;20:859-868.

23. Silver JK. Cancer prehabilitation: an opportunity to decrease treatment-related morbidity, increase cancer treatment options, and improve physical and psychological health outcomes. Am J Phys Med Rehabil. 2013;92:715-727.

24. Demark-Wahnefried W, Peterson B, McBride C, Lipkus I, Clipp EJ. Current health behaviors and readiness to pursue life-style changes among men and women diagnosed with early stage prostate and breast carcinomas. Cancer. 2000;88:674-684.

25. Alderman G, Semple S, Cesnik R, Toohey K. Health care professionals' knowledge and attitudes toward physical activity in cancer patients: a systematic review. Semin Oncol Nurs. 2020;36: 151070.

26. Bultijnck R, Surcel C, Ploussard G, et al. Practice patterns compared with evidencebased strategies for the management of androgen deprivation therapy-induced side effects in prostate cancer patients: results of a European web-based survey. Eur Urol Focus. 2016;2:514-521.

27. Nyrop KA, Deal AM, Williams GR, Guerard EJ, Pergolotti M, Muss HB. Physical activity communication between oncology providers and patients with early-stage breast, colon, or prostate cancer. Cancer. 2016;122:470-476

28. Hardcastle SJ, Kane R, Chivers P, et al. Knowledge, attitudes, and practice of oncologists and oncology health care providers in promoting physical activity to cancer survivors: an international survey. Supp Care Cancer. 2018;26:3711-3719.

29. Galvão DA, Newton RU, Gardiner RA, et al. Compliance to exercise-oncology guidelines in prostate cancer survivors and associations with psychological distress, unmet supportive care needs, and quality of life. Psychooncology. 2015;24:1241-1249.

30. Zopf EM, Newton RU, Taaffe DR, et al. Associations between aerobic exercise levels and physical and mental health outcomes in men with bone metastatic prostate cancer: a cross-sectional investigation. Eur J Cancer Care (Engl). 2017;26:e12575.

31. Blanchard CM, Courneya KS, Stein KJ. Cancer survivors' adherence to lifestyle behavior recommendations and associations with health-related quality of life: results from the American Cancer Society's SCS-II. J Clin Oncol. 2008;26:2198-2204.

32. Karvinen KH, Courneya KS, North S, Venner PJCE, Biomarkers P. Associations between exercise and quality of life in bladder cancer survivors: a populationbased study. Cancer Epidemiol Biomarkers Prev. 2007;16:984-990.

33. Gopalakrishna A, Longo TA, Fantony JJ, Harrison MR, Inman BA. Physical activity patterns and associations with health-related quality of life in bladder cancer survivors. Urol Oncol. 2017;35. 540. e541-540. e546.

34. Trinh L, Strom DA, Wong JN, Courneya KSJPo. Modality-specific exercise guidelines and quality of life in kidney cancer survivors: a cross-sectional study. Psychooncology. 2018;27:2419-2426.

35. Ntoumanis N, Thørgersen-Ntoumani C, Quested E, Chatzisarantis N. Theoretical approaches to physical activity promotion. Oxford Research Encyclopedia of Psychology. 2018. https://doi.org/10.1093/acrefore/9780190236557.013.212. Available at: Accessed Month 10, 2020

36. Grimmett C, Corbett T, Brunet J, et al. Systematic review and meta-analysis of maintenance of physical activity behaviour change in cancer survivors. Int J Behav Nutr Phys Act. 2019;16:37.

37. Short C, James EL, Plotnikoff RC. How social cognitive theory can help oncologybased health professionals promote physical activity among breast cancer survivors. Eur J Oncol Nurs. 2013;17:482-489.

38. Brug J, Oenema A, Ferreira IJ. Theory, evidence and intervention mapping to improve behavior nutrition and physical activity interventions. Int I Behav Nutr Phys Act. 2005;2:2.

39. Finne E, Glausch M, Exner AK, et al. Behavior change techniques for increasing physical activity in cancer survivors: a systematic review and meta-analysis of randomized controlled trials. Cancer Manage Res. 2018;10:5125

40. Dombrowski SU, O'Carroll RE, Williams B. Form of delivery as a key “active ingredient" in behaviour change interventions. Br J Health Psychol. 2016;21:733-740.

41. McEwan D, Beauchamp MR, Kouvousis C, Ray CM, Wyrough A, Rhodes RE. Examining the active ingredients of physical activity interventions underpinned by theory versus no stated theory: a meta-analysis. Health Psychol Rev. 2019;13:1-17.

42. Troeschel AN, Leach CR, Shuval K, Stein KD, Patel AV. Physical activity in cancer survivors during "re-entry" following cancer treatment. Prev Chronic Dis. 2018;15:E65.

43. Ajzen I. The theory of planned behaviour: reactions and reflections. Psychol Health. 2011;26:1113-1127. 
44. Pinto BM, Ciccolo JT. Physical activity motivation and cancer survivorship. Recent Results Cancer Res. 2011;186:367-387.

45. Thorsen L, Courneya KS, Stevinson C, Fosså SD. A systematic review of physical activity in prostate cancer survivors: outcomes, prevalence, and determinants. Support Care Cancer. 2008;16:987-997.

46. Courneya KS, Segal RJ, Reid RD, et al. Three independent factors predicted adherence in a randomized controlled trial of resistance exercise training among prostate cancer survivors. J Clin Epidemiol. 2004;57:571-579.

47. Prochaska JO, Redding CA, Evers KE. The transtheoretical model and stages of change In K. Glanz, B. K. Rimer, \& K. "V." Viswanath (Eds.), Health behavior: Theory, research, and practice. Hoboken, New Jersey: Jossey-Bass/Wiley; 2015:125-148.

48. Prochaska JO, Marcus BH. The transtheoretical model: applications to exercise. Med Sci Sports Exerc. 1994:26:1400-1404.

49. Rammant E, Fonteyne V, Decaestecker K, et al. Understanding physical activity behavior in patients with bladder cancer before and after radical cystectomy: a qualitative interview study. Clin Rehab. 2019;33:750-761.

50. Trinh L, Larsen K, Faulkner GE, et al. Social-ecological correlates of physical activity in kidney cancer survivors. J Cancer Surviv. 2016;10:164-175.

51. Finlay A, Wittert G, Short CE. A systematic review of physical activity-based behaviour change interventions reaching men with prostate cancer. J Cancer Surviv. 2018;12:571-591.

52. Bartholomew LK, Parcel GS, Kok GJ. Intervention mapping: a process for developing theory and evidence-based health education programs. Health Educ Behav. 1998;25:545-563.

53. Bandura AJHop. Social cognitive theory of personality. Annu Rev Psychol. 1999;2: $154-196$.

54. Trinh L, Kramer AF, Rowland K, Strom DA, Wong JN, McAuley E. A pilot feasibility randomized controlled trial adding behavioral counseling to supervised physical activity in prostate cancer survivors: behavior change in prostate cancer survivors trial (BOOST) [published online ahead of print]. J Behav Med. 2020.

55. Rhodes RE. The evolving understanding of physical activity behavior: a multi-process action control approach. Adv Motiv Sci. 2017;4:171-205.

56. Hallward L, Patel N, Duncan LR. Behaviour change techniques in physical activity interventions for men with prostate cancer: a systematic review. J Health Psychol. 2020;25:105-122

57. Rammant E, Deforche B, Van Hecke A, et al. Development of a pre- and postoperative physical activity promotion program integrated in the electronic health system of patients with bladder cancer (The POPEYE study): an intervention mapping approach. Eur J Cancer Care. 2020:e13363.

58. Jensen BT, Petersen AK, Jensen JB, Laustsen S, Borre MJ. Efficacy of a multiprofessional rehabilitation programme in radical cystectomy pathways: a prospective randomized controlled trial. Scand J Urol. 2015;49:133-141.

59. Trinh L, Plotnikoff RC, Rhodes RE, North S, Courneya KS. Feasibility and preliminary efficacy of adding behavioral counseling to supervised physical activity in kidney cancer survivors: a randomized controlled trial. Cancer Nurs. 2014;37:E8-E22.
60. Trinh L, Plotnikoff RC, Rhodes RE, North S, Courneya KS. Changes in motivational outcomes following a supervised physical activity program with behavioral counseling in kidney cancer survivors: a pilot study. Psychooncology. 2015;24: 1204-1207.

61. Fernandez ME, Ruiter RA, Markham CM, Kok GJ. Theory-and evidence-based health promotion program planning: intervention mapping. Front Public Health. 2019;7:209.

62. Coole C, Baker P, McDaid C, Drummond A. Using intervention mapping to develop an occupational advice intervention to aid return to work following hip and knee replacement in the United Kingdom. BMC Health Serv Res. 2020;20:523.

63. Craig P, Dieppe P, Macintyre S, Michie S, Nazareth I, Petticrew M. Developing and evaluating complex interventions: the new Medical Research Council guidance. BMJ. 2008;337:a1655.

64. Golsteijn R, Bolman C, Volders E, Peels D, de Vries H, Lechner L. Development of a computer-tailored physical activity intervention for prostate and colorectal cancer patients and survivors: OncoActive. BMC Cancer. 2017; 17:446.

65. Evans HEL, Forbes CC, Galvão DA, et al. Evaluating a web- and telephone-based personalised exercise intervention for individuals living with metastatic prostate cancer (ExerciseGuide): protocol for a pilot randomised controlled trial. Pilot Feasibility Stud. 2021;7:21. https://doi.org/10.1186/s40814-020-00763-2.

66. Rammant E, Deforche B, Van Hecke A, et al. Development of a pre- and postoperative physical activity promotion program integrated in the electronic health system of patients with bladder cancer (The POPEYE study): an intervention mapping approach. Eur J Cancer Care. 2020:e13363.

67. Davis R, Campbell R, Hildon Z, Hobbs L, Michie S. Theories of behaviour and behaviour change across the social and behavioural sciences: a scoping review. Health Psychol Rev. 2015;9:323-344.

68. Michie S, Ashford S, Sniehotta FF, et al. A refined taxonomy of behaviour change techniques to help people change their physical activity and healthy eating behaviours: the CALO-RE taxonomy. Psychol Health. 2011;26:1479-1498.

69. Baranowski T, Anderson C, Carmack C. Mediating variable framework in physica activity interventions: How are we doing? How might we do better? Am J Prevent Med. 1998;15:266-297.

70. Bauman AE, Sallis JF, Dzewaltowski DA, Owen N. Toward a better understanding of the influences on physical activity: the role of determinants, correlates, causal variables, mediators, moderators, and confounders. Am J Prevent Med. 2002;23: 5-14.

71. Rhodes RE, Boudreau P, Josefsson KW, Ivarsson A. Mediators of physical activity behaviour change interventions among adults: a systematic review and metaanalysis [published online ahead of print]. Health Psychol Rev. 2020.

72. Noar SM, Zimmerman RS. Health behavior theory and cumulative knowledge regarding health behaviors: are we moving in the right direction? Health Educ Res $2005 ; 20: 275-290$ 